\title{
$A C$ Loss Measurements of a Trapezoidal Shaped HTS Coil Using an Electrical Method
}

\author{
G. Messina, ${ }^{1}$ L. Morici, ${ }^{1}$ U. Besi Vetrella, ${ }^{1}$ G. Celentano, ${ }^{1}$ M. Marchetti, ${ }^{1}$ \\ R. Viola, ${ }^{1}$ and P. Sabatino ${ }^{2}$ \\ ${ }^{1}$ ENEA, Unità Tecnica Fusione, ENEA C. R. Frascati, Via Enrico Fermi 45, Frascati, 00044 Roma, Italy \\ ${ }^{2}$ Università di Salerno, Via Giovanni Paolo II 132, Fisciano, 84084 Salerno, Italy \\ Correspondence should be addressed to G. Messina; giuseppe.messina.fra@enea.it and L. Morici; luigi.morici@enea.it
}

Received 31 July 2014; Revised 11 November 2014; Accepted 12 November 2014; Published 2 December 2014

Academic Editor: Dong Qian

Copyright (C) 2014 G. Messina et al. This is an open access article distributed under the Creative Commons Attribution License, which permits unrestricted use, distribution, and reproduction in any medium, provided the original work is properly cited.

\begin{abstract}
High temperature superconducting (HTS) coils of different shapes (typically circular or trapezoidal) wound on iron or ironless core are fundamental components of many superconducting electrical power devices. A 150-turn (75 turns/pancake) trapezoidal coil in double pancake configuration has been designed and realized in our laboratory of ENEA Frascati. Various epoxy resins and YBCO tapes have been tested in the temperature range room to liquid nitrogen, leading us to the choice of AmSC (American Superconductor) tape for the winding and araldite resin for the impregnation process. The trapezoidal shape has been chosen because of its suitable geometry for practical applications, the results being complementary to what was previously achieved on round shaped HTS coils. The $A C$ transport current losses have been measured using a compensated electrical method and expressed in terms of a linearly frequency dependent resistance. A linear dependence of the losses resistance from frequency was expected and found in agreement with previous results. The current-voltage curve has been measured in zero externally applied field condition, the results being in good agreement with a numerical simulation. The magnetic field distributions, at different air gaps from coil top and zero externally applied filed condition, have been simulated and reported as well.
\end{abstract}

\section{Introduction}

Second generation (2G) high temperature superconducting (HTS) coils have a range of applications in electrical devices, such as superconducting fault current limiter (SFCL), superconducting magnetic energy storage (SMES), and superconducting generators and motors. The zero resistance of any superconducting $(S C)$ material is only observed in $D C$ conditions; in an $A C$ environment, any varying magnetic field interacts with the $S C$ material giving rise to energy dissipation: AC transport current losses if the magnetic field is self-generated and $A C$ magnetization losses if the magnetic field is externally applied. $A C$ losses give rise to a thermal load for the cryogenic system, resulting in a constraint for the use and operation of superconducting materials and hard measures of interest in this paper. Critical currents, air-gap magnetic flux density, and $A C$ losses analysis of HTS coils components are important steps in complex devices design, determining the application ranges of the rated currents and magnetic fields of superconducting material. $A C$ losses, being responsible of the cryogenic equipment required power, are among the greatest factors influencing the overall economic impact of SC devices. To increase air-gap magnetic flux density in axial flux electrical machines, various shapes of HTS coils have been manufactured and tested in our facilities. Cylindrical coils made with commercial coated conductors have been largely studied in several works, either by measurements and/or computations [1-10], while the trapezoidal shape is taken into account here to optimize the statorrotor system coupling. In this paper, a complete theoretical and experimental study of a trapezoidal shape $2 \mathrm{G}$ HTS coil is reported. The paper is structured as follows: double pancake HTS coil manufacturing process presentation, liquid nitrogen temperature of the coil critical currents presentation and comparison with a FEM model simulation, an electrical measurement method description for the $A C$ condition, $A C$ transport current losses results expressed in terms of an equivalent frequency dependent loss resistance, discussion, and final conclusions. 


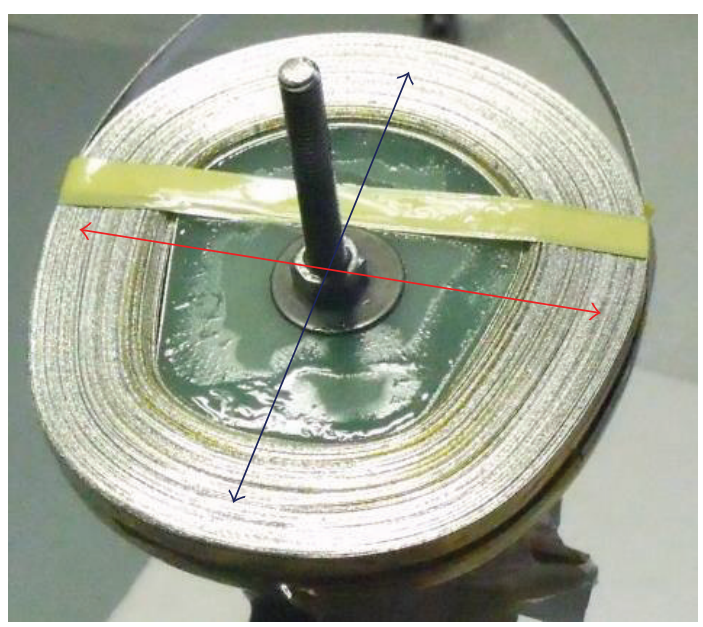

(a)

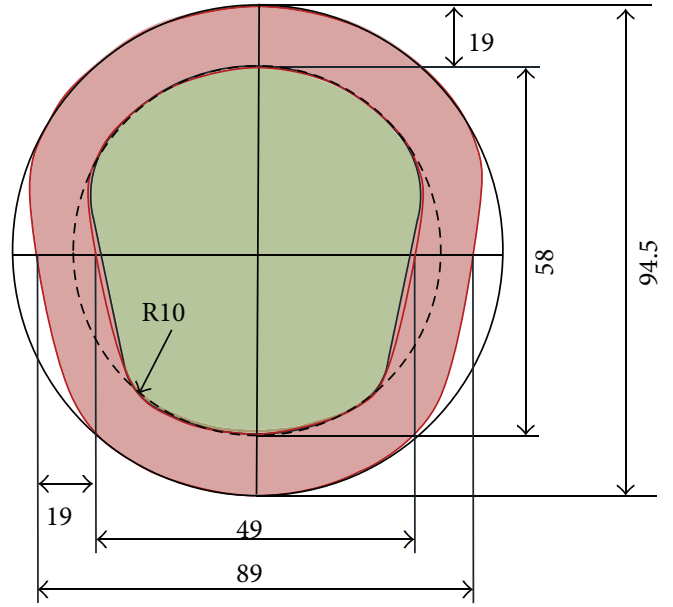

(b)

Figure 1: (a) Trapezoidal shape HTS coil. (b) All dimensions in $\mathrm{mm}$ : height $=13 \mathrm{~mm}$, maximum bending $=10 \mathrm{~mm}$.

\section{Trapezoidal Coil Description}

To manufacture our trapezoidal coil, we used a $(4.8 \mathrm{~mm} \times$ $0.2 \mathrm{~mm}$ ) magnetic substrate YBCO copper laminated certified Amperium tape, manufactured by American Superconductor (AmSC). The tape electrical self-field critical current is $I_{c}>100 \mathrm{~A}$ at $77 \mathrm{~K}$. About $35 \mathrm{~m}$ of the Amperium tape has been used to wind a 150-turn (75 turns per pancake) double pancake coil around a PVC trapezoidal shaped core, side by side. To avoid tape damages during bending, the core has been designed considering the tape minimum acceptable curvature radius. The winding process was carried out at room temperature, starting at midsection, insulating each wind with a Kapton tape, and stretching the tape with $1 \mathrm{~N}$ constant force, the force being applied to avoid any liftoff issue around the corners. During HTS tape winding, the YBCO layer was outward oriented with an insulating tape between adjacent turns. Once the winding process has been carried out, an impregnation material (epoxy resin a.k.a. araldite) has been applied to the coil. Finally, the first and last turns have been soldered to current leads [11]. The impregnation material has been treated according to the manufacturer preparation specifications; curing was not performed, the samples being dried at room temperature. Voltage taps have been soldered at coil ends for overall voltage measurement. The HTS coil is pictured in Figure 1(a), geometric parameters being the following: larger size (blue line) $D=94.5 \mathrm{~mm}$, smaller size (red line) $d=89 \mathrm{~mm}$, height $h=13 \mathrm{~mm}$, winding thickness $=19 \mathrm{~mm}$.

\section{Critical Current Measurement and Simulation}

To estimate the critical current of our HTS coil, the currentvoltage characteristic at zero external field condition has been measured using the 4 probes standard electrical method. The $1 \mu \mathrm{Vcm}^{-1}$ criteria threshold has been used for $I_{c}$ determination; as shown in Figure 2(a), we measured $I_{c}=55.6 \mathrm{~A}$.
For simulation purposes, the coil trapezoidal shape has been approximated with a circular shape (Figure 1(b)) [12]. Accordingly, a two-dimensional (2D) axial symmetrical FEM model for the coil cross section, based on the $H$-formulation, has been worked out using the software package COMSOL Multiphysics. With the usual meaning of the symbols, Faraday's and Ampere's laws $\left(\nabla \times \mathbf{E}=-\partial \mathbf{B} / \partial \mathbf{t}, \nabla \times \mathbf{B}=\mu_{0} \mathbf{J}\right)$, as well as the material constitutive laws $\left(\mathbf{B}=\mu_{0} \mu_{r} \mathbf{H}, \mathbf{E}=\rho \mathbf{J}\right)$, have been implemented. In the $2 \mathrm{D}$ approximation, we use $(r, z)$ cylindrical coordinate for the coil cross section in order to have only two variables in the $H$-formulation; that is, $\mathbf{H}=$ $\left(H_{r}, H_{z}\right)$. The current $J_{\phi}$ of the coil flows in the $\phi$ direction generating an azimuthal electric field, $E=E_{\phi}=\rho \cdot J_{\phi}$, while Ampere and Faraday's laws become, respectively,

$$
\begin{gathered}
J_{\phi}=\nabla \times \mathbf{H}=\frac{\partial H_{r}}{\partial z}-\frac{\partial H_{z}}{\partial r} \\
\nabla \times \mathbf{E}=\left(\begin{array}{c}
-\frac{\partial E_{\phi}}{\partial z} \\
\frac{1}{r} \frac{\partial\left(r E_{\phi}\right)}{\partial r}
\end{array}\right)=-\mu_{0}\left(\begin{array}{c}
\frac{\partial\left(\mu_{r} H_{r}\right)}{\partial t} \\
\frac{\partial\left(\mu_{r} H_{z}\right)}{\partial t}
\end{array}\right) .
\end{gathered}
$$

The ReBCO tape has been modeled using real thicknesses: $1 \mu \mathrm{m}$ for YBCO layers and $75 \mu \mathrm{m}$ for magnetic substrate layers with $0.3 \mu \mathrm{m}$ separation [13]. The E-J law has been defined separately for the superconducting layer, where it is mainly nonlinear, from the other materials where resistivity $(\rho)$ is a constant:

$\rho(B, \theta)$

$=\left\{\begin{array}{l}10^{5} \Omega \mathrm{m} \\ \frac{E_{c}}{J_{c}(B, \theta)} \\ 5 \mu \Omega \mathrm{m}\end{array} \cdot\left(\frac{J_{\phi}}{J_{c}(B, \theta)}\right)^{(n(B, \theta)-1)}\right.$

(air)

(magnetic substrate), 


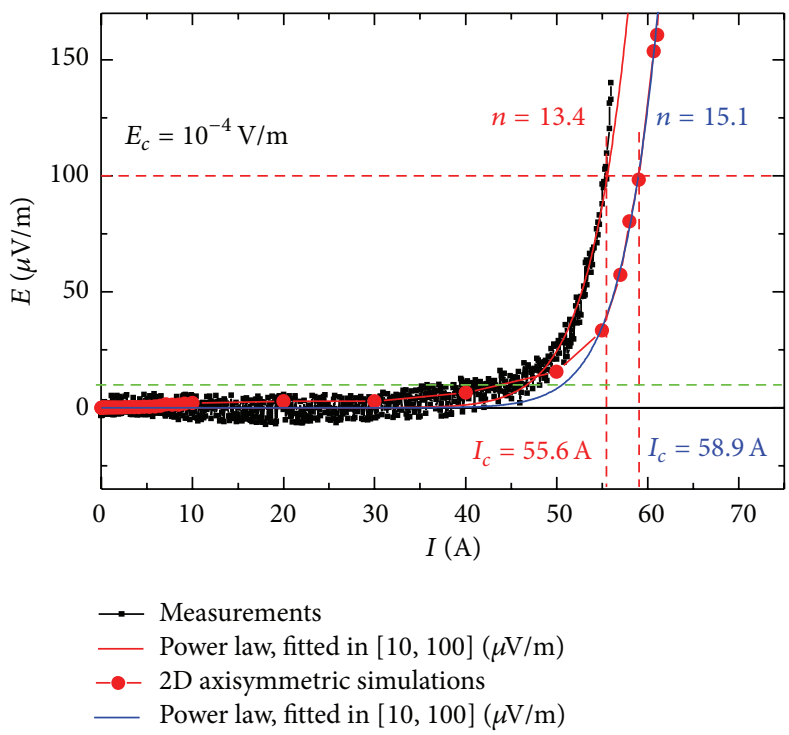

(a)

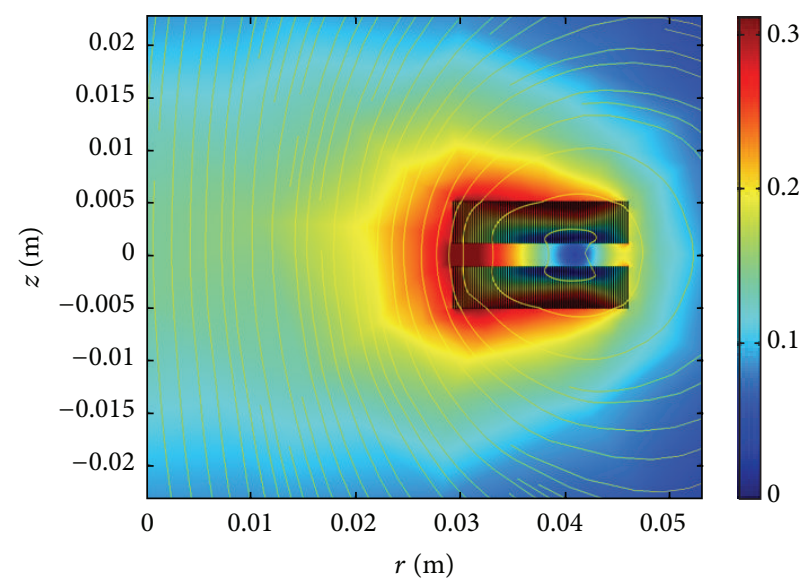

(b)

Figure 2: (a) Comparison of $I V$ curve at $77 \mathrm{~K}$ measured and numerically calculated. (b) Snapshot of magnetic field intensity $B$ (T) computed at the critical current $I_{c}=59 \mathrm{~A}$.

$\theta \equiv$ angle between tape $c$-axis (i.e., current direction) and magnetic induction B. AmSC tape characteristic properties have been used, this way improving simulated and measured data agreement; in particular, an elliptical composition of parallel and perpendicular behavior, of both the critical current and the $n$-value, has been introduced for the anisotropic YBCO layer modeling $[1,11,14]$. The nonlinear $B-H$ law of the substrate material (Ni-5at\%W) has been implemented in the FEM code defining the relative magnetic permeability as a function of magnetic field [15], while we used a unitary permeability in the remaining materials:

$$
\begin{aligned}
& \mu_{r}(H) \\
& =\left\{\begin{array}{cc}
1 & \text { (HTS and air) } \\
1+30600\left(1-\exp \left(-\left(\frac{H}{295}\right)^{2.5}\right)\right) & \\
\times H^{-0.81}+45 \exp \left(-\left(\frac{H}{120}\right)^{2.5}\right) & \text { (substrate) } .
\end{array}\right.
\end{aligned}
$$

A code computed $I-V$ curve of the double pancake coil has been carried out to test the model. In the $2 \mathrm{D}$ simulation, the current is ramped up to a value $I$ at $f=0.02 \mathrm{~Hz}$ and then kept constant for $1 \mathrm{~s}$ (in order to relax the calculated voltage). As can be seen in Figure 2(a), the experimental $I_{c}=55.6 \mathrm{~A}$ is roughly consistent with the simulated $I_{c}=$ $58.9 \mathrm{~A}$, discrepancies being due to coil section flattening along the radial coordinate (Figure $1(\mathrm{~b})$ ) producing a magnetic field strength higher than the corresponding cylindrical configuration used in the simulation. Our model is validated from the quite good approximation just described (only about $6 \%$ error on $I_{c}$ ), opening the possibility for $A C$ losses estimation. Finally, the total magnetic field in coil critical current condition is shown in Figure 2(b). Experimental and simulated results are in good agreement and can be useful for the design of superconducting axial flux electrical machines (AFEM). In this regard, it is important to know the axial and radial profiles of the air-gap magnetic flux density for different current values. In particular, the radial magnetic field distributions are useful to get estimations of the $A C$ losses of a coil when the transport current is at peak value $I[3,4]$. The calculated results are briefly summarized in Figure 3.

\section{AC Transport Current Losses}

4.1. Experimental Apparatus. A sketch of our measurement setup is shown in Figure 4. A nonmetallic cryostat (not shown in the picture) keeps the superconducting coil, $Z_{\text {coil }}$, at liquid nitrogen temperature. As expected, no impedance comes from the coil at zero frequency, the impedance rising as soon as any frequency dependent driving signal is applied to the $S C$ coil. The $Z_{\text {coil }}(f)$ contains an undesired imaginary component coming from the device inductance $\left(L_{\text {coil }}=\right.$ $2.06 \mathrm{mH}$ ) and a real component which, being produced from $A C$ losses, is the measure of our interest. To measure the $Z_{\text {coil }}(f)$ components we used a lock-in amplifier. Besides, to maximize SNR, a compensation technique of the undesired imaginary part of the signal has been implemented. Our compensation circuitry is made of a capacitors bank connected in series to the $S C$ coil, the bank capacitance being varied during the experiment in order to keep an acceptable SNR for a given measurement frequency. Finally, part of our experimental setup is at liquid nitrogen temperature (the $S C$ coil) and part is at room temperature (the compensating capacitors and a portion of the connection cables). Particular care is required for circuitry parameters estimation, being the real part of the 


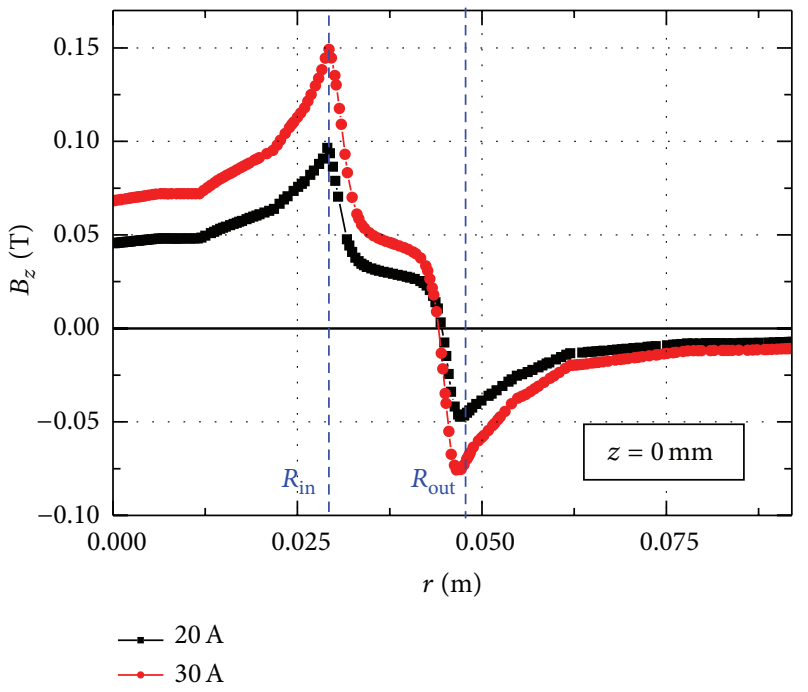

(a)

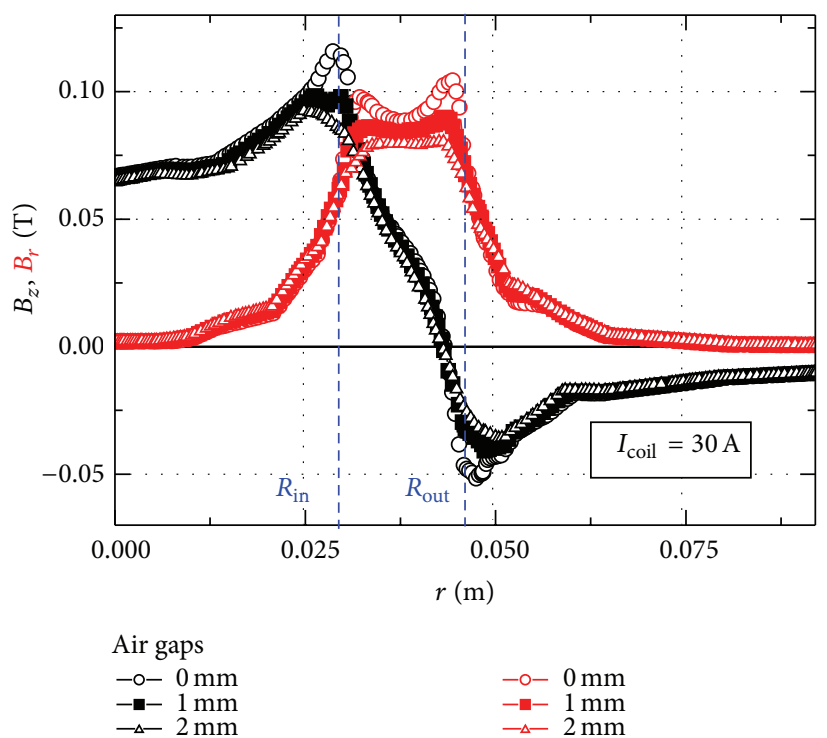

(b)

FIgURE 3: (a) Comparison of computed axial component of $B$ as a function of distance from magnetic axis of the coil for different current values. (b) Computed axial and radial components of $B$ as a function of distance from magnetic axis of the coil for different air gaps at $I_{\text {coil }}=30 \mathrm{~A}$.

impedance we are interested in, in a few milliohm range (i.e., of the order of the copper connection cables resistance of our circuit), and for that reason we used a precision LCR meter to measure the bank capacitance at each frequency value. Accordingly, the two T switches in Figure 4 are there to avoid any circuitry configuration changes between measures. To generate an ohmic reference signal $\left(V_{\text {shunt }}\right)$, a $40 \mathrm{~m} \Omega$ shunt resistance $\left(R_{\text {shunt }}\right)$ has been included in the setup. The $V_{\text {total }}$ and $V_{\text {shunt }}$ signals have been connected at lock-in $\mathrm{CH}-\mathrm{A}$ and Ref, respectively. For given current and frequency values, the expressions reported in the following set of equations,

$$
\begin{gathered}
Z_{\text {coil }}=R_{\text {loss }}+j \omega L_{\text {coil }}, \\
V_{\text {total }}=\left(Z_{\text {coil }}+Z_{\text {cable }}+Z_{c}\right) \cdot I \\
=\left[\left(R_{\text {loss }}+R_{\text {cable }}+R_{c}\right)+j\left(\omega \cdot L_{\text {coil }}-\frac{1}{\omega \cdot C}\right)\right] \cdot I \\
\operatorname{Re}\left(V_{\text {total }}\right)=\left(R_{\text {loss }}+R_{\text {cable }}+R_{c}\right) \cdot I,
\end{gathered}
$$

have been used in our analysis to compute the coil resistance $\left(R_{\text {loss }}\right)$, which is the parameter of our interest (being directly linked to the superconductor $A C$ losses), while the chosen bank of capacitors configuration is reported as follows.

Bank Capacitors Configuration. Consider the following:

(1) $C_{1}=45 \mathrm{mF}$ and $C_{2}=45 \mathrm{mF}$ connected in parallel;

(2) $C_{1}=45 \mathrm{mF}$ and $C_{2}=45 \mathrm{mF}$ connected in series;

(3) $C_{1}=45 \mathrm{mF}$.
4.2. Experimental Results. The high $Q$ value of the resonant circuit of our experimental apparatus determines, for a given capacitance, a small range of frequency values measurable with optimum SNR. As a result, the compensation impedance $Z_{c}$ has to be varied in order to obtain the frequency span we are interested in, this last requirement being fulfilled using a capacitance bank. A first step to estimate the $A C$ transport current losses in the $S C$ coil is to measure the current in phase component of the overall voltage across the capacitance-coil impedance series $\left(\operatorname{Re}\left(V_{\text {total }}\right)\right)$. By subtracting the $R_{c}$ contribution as obtained from the LCR meter, a measure of the voltage drop across the coil-copper connection cable series $\left(R_{\text {loss }}+R_{\text {cable }}\right)$ is obtained. The expected behavior of $R_{\text {loss }}$ is a frequency dependent contribution due to the superconductor tape, plus a constant bias coming from the copper connection cables. As is well known [16], $R_{\text {loss }}(f)$ has a linear dependence with frequency whenever the copper tape conducting matrix loss contribution is negligible $\left(R_{\mathrm{Cu}}\right)$. In this case, a good description of the system behavior is given by the expression: $R_{\text {loss }}(f, I)+R_{\text {cable }}=R_{0}(I) \cdot f+R_{\text {cable }}$ (the frequency dependence of the copper matrix being quadratic, when ratable). $R_{\text {cable }}$ is the undesired series resistance component of the cables connecting the liquid nitrogen $S C$ coil to the room temperature $\mathrm{T}$ switch. Our experimental results for $R_{\text {loss }}(f)+R_{\text {cable }}$ are reported in Figure 5 for three driving rms current values. As can be seen, the common value of the zero frequency intercept of each line gives the connection cable resistance of our experimental setup $\left(R_{\text {cable }}=6 \mathrm{~m} \Omega\right.$ in our case), while the slope of each line gives the $R_{0}(I)$ parameter. The current dependence of $R_{0}(I)$ is due to transport current losses associated with the coil self-produced magnetic field and is coil geometric configuration dependent. As can be seen 


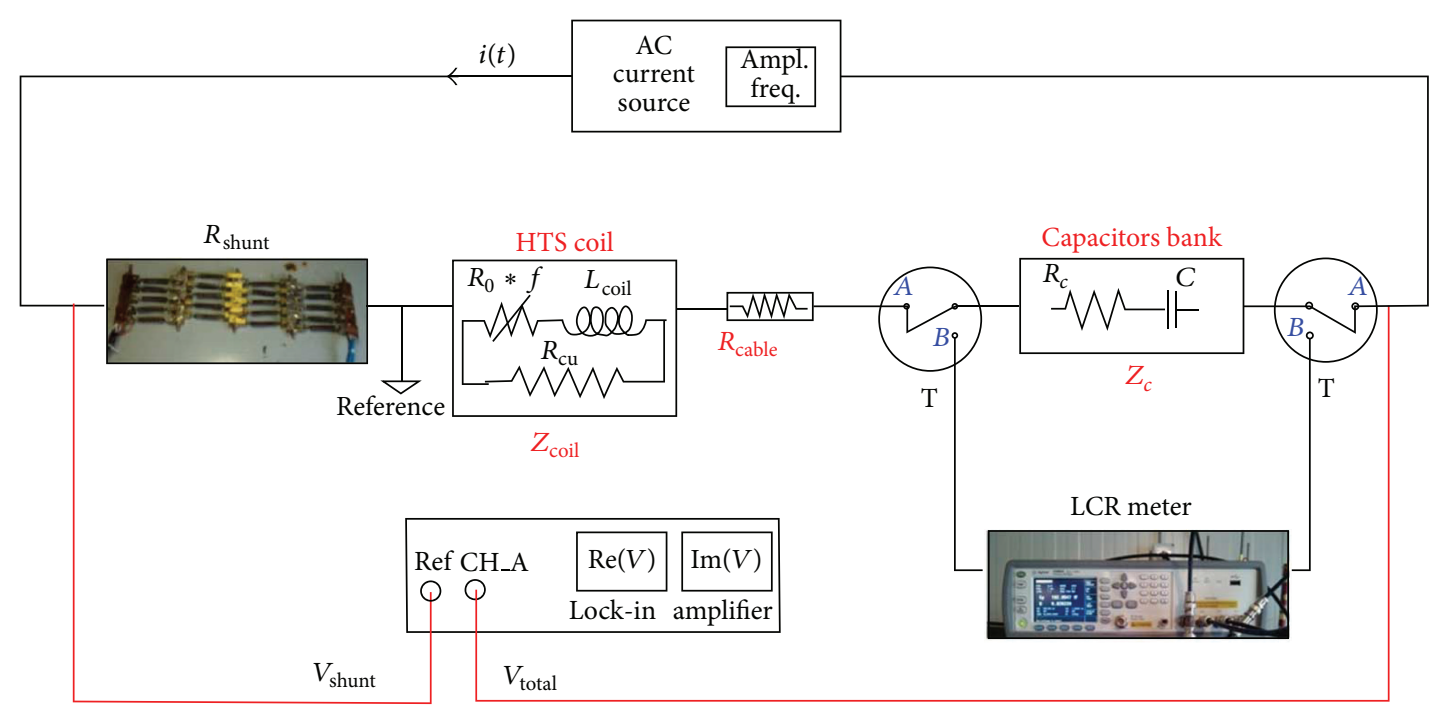

FIGURE 4: Electrical circuit for $A C$ losses measurements.

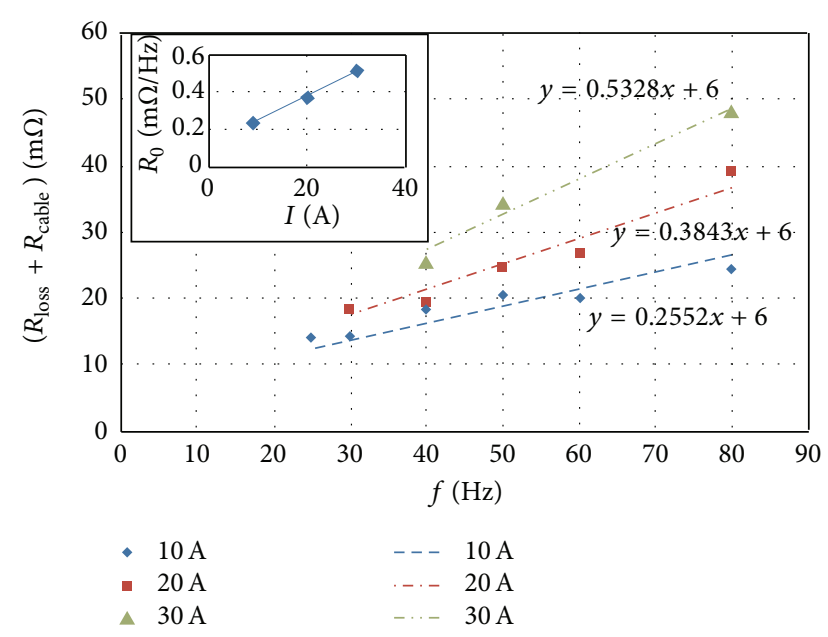

Figure 5: $R_{\text {loss }}$ versus frequency at various rms current values; in the insert, lines slope evolution with current.

in the insert in Figure 5, we obtained a linear dependence of $R_{0}$ versus rms $I$ in our case.

\section{Conclusions}

We presented the measured and simulated data on a trapezoidal HTS coil in double pancake configuration. In the DC condition, critical current measured and simulated results were in good agreement. To study the $A C$ superconducting coil behavior, we settled up an experimental apparatus based on reactance compensation. Within the analyzed frequency range $(30-80 \mathrm{~Hz})$, we found a negligible contribution of the eddy current losses induced on the superconductor copper matrix. Finally, we identified a single parameter, namely, $R_{0}$, that, for a given SC tape and geometric configuration of the superconducting component being analyzed (a trapezoidal coil in our study), may be used to estimate the superconductor contribution to the overall transport current losses, making $R_{0}$ a really useful design parameter.

\section{Conflict of Interests}

The authors declare that there is no conflict of interests regarding the publication of this paper.

\section{References}

[1] M. D. Ainslie, V. M. Rodriguez-Zermeno, Z. Hong, W. Yuan, T. J. Flack, and T. A. Coombs, "An improved FEM model for computing transport AC loss in coils made of RABiTS YBCO coated conductors for electric machines," Superconductor Science and Technology, vol. 24, no. 4, Article ID 045005, 2011.

[2] F. Gömöry, J. Šouc, E. Pardo et al., "AC loss in pancake coil made from $12 \mathrm{~mm}$ wide REBCO tape," IEEE Transactions on Applied Superconductivity, vol. 23, no. 3, Article ID 5900406, 2013.

[3] W. Yuan, M. D. Ainslie, W. Xian et al., "Theoretical and experimental studies on Jc and AC losses of 2G HTS coils," IEEE Transactions on Applied Superconductivity, vol. 21, no. 3, pp. 2441-2444, 2011.

[4] W. Yuan, A. M. Campbell, Z. Hong, M. D. Ainslie, and T. A. Coombs, "Comparison of AC losses, magnetic field/current distributions and critical currents of superconducting circular pancake coils and infinitely long stacks using coated conductors," Superconductor Science and Technology, vol. 23, no. 8, Article ID 085011, 2010.

[5] F. Grilli and S. P. Ashworth, "Measuring transport AC losses in YBCO-coated conductor coils," Superconductor Science and Technology, vol. 20, no. 8, pp. 794-799, 2007.

[6] E. Pardo, J. Ŝouc, and J. Kovác, "AC loss in ReBCO pancake coils and stacks of them: modelling and measurement," Superconductor Science and Technology, vol. 25, no. 3, Article ID 035003, 2012.

[7] V. Grinenko, G. Fuchs, K. Nenkov et al., "Transport AC losses of YBCO pancake coils wound from parallel connected tapes," 
Superconductor Science and Technology, vol. 25, no. 7, Article ID 075006, 2012.

[8] M. Zhang, J. Kvitkovic, S. V. Pamidi, and T. A. Coombs, "Experimental and numerical study of a YBCO pancake coil with a magnetic substrate," Superconductor Science and Technology, vol. 25, no. 12, Article ID 125020, 2012.

[9] M. Zhang, J. Kvitkovic, J.-H. Kim, C. H. Kim, S. V. Pamidi, and T. A. Coombs, "Alternating current loss of second-generation high-temperature superconducting coils with magnetic and non-magnetic substrate," Applied Physics Letters, vol. 101, Article ID 102602, 2012.

[10] M. Polak, E. Demencik, L. Jansak et al., "Ac losses in a $Y \mathrm{Ba}_{2}$ $\mathrm{Cu}_{3} \mathrm{O}_{7-x}$ coil," Applied Physics Letters, vol. 88, no. 23, Article ID 232501, 2006.

[11] U. Besi Vetrella, G. Celentano, M. Marchetti et al., "HTS coils fabrication from commercial 2G YBCO tapes: measurements and simulation," IEEE Transactions on Applied Superconductivity, vol. 24, no. 3, Article ID 4600204, 2014.

[12] G. Messina, L. Morici, U. Besi Vetrella et al., "Modelling and measurements of circular and trapezoidal shape HTS coils for electrical machines applications," Journal of Physics: Conference Series, vol. 507, Article ID 032031, 2014.

[13] A. Malozemoff, M. Rupich, and U. Schoop, "Scale-up of Second Generation HTS Wire (2G-YBCO Coated Conductor)," DOE Peer Review, 2004.

[14] G. Messina, L. Morici, U. Besi Vetrella et al., "AC transport current losses in HTS coils for axial flux electrical machines applications," IEEE Transactions on Applied Superconductivity, vol. 24, no. 3, Article ID 4602204, 2014.

[15] D. N. Nguyen, S. P. Ashworth, J. O. Willis, F. Sirois, and F. Grilli, "A new finite-element method simulation model for computing AC loss in roll assisted biaxially textured substrate YBCO tapes," Superconductor Science and Technology, vol. 23, no. 2, Article ID 025001, 2010.

[16] C. M. Magro, M. Neves, A. Sfetsos, J. Pina, and A. Gonçalves, "Multipole superconducting synchronous generator," in Proceedings of the 6th European Conference on Applied Superconductivity (EUCAS '03), 2003. 

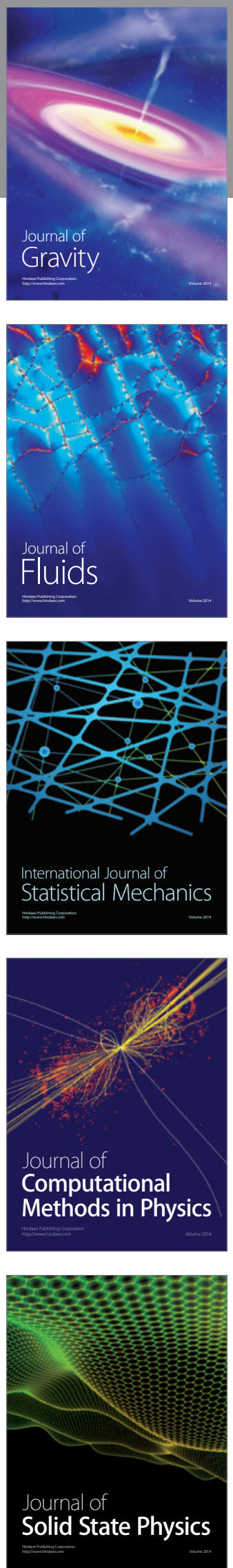

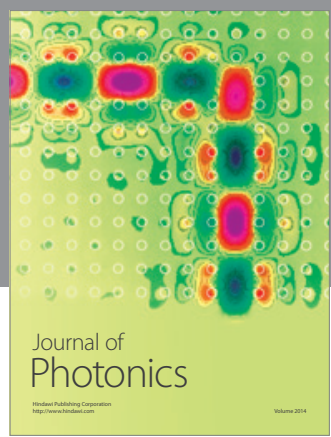

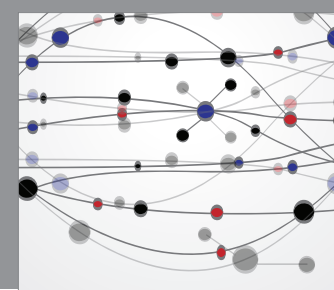

The Scientific World Journal

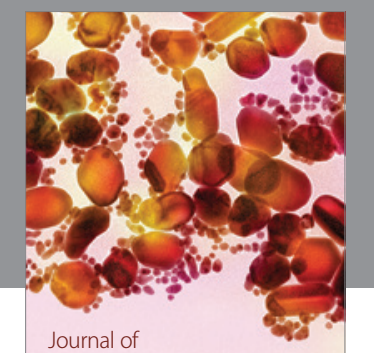

Soft Matter
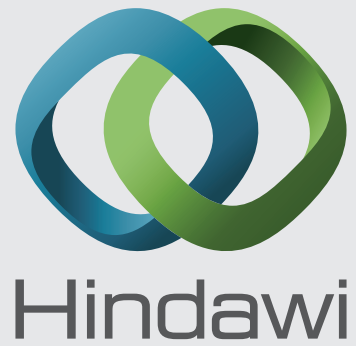

Submit your manuscripts at

http://www.hindawi.com
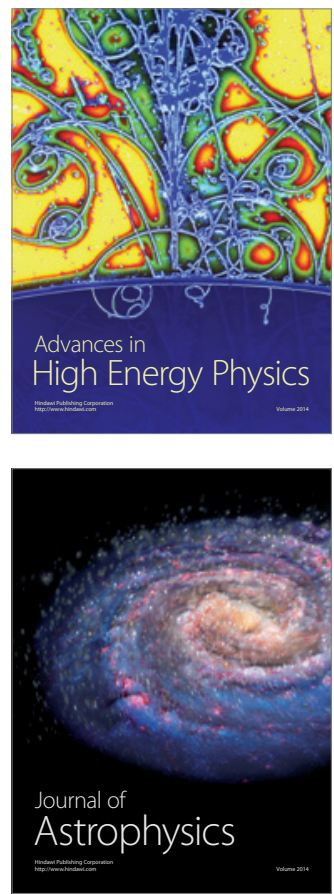
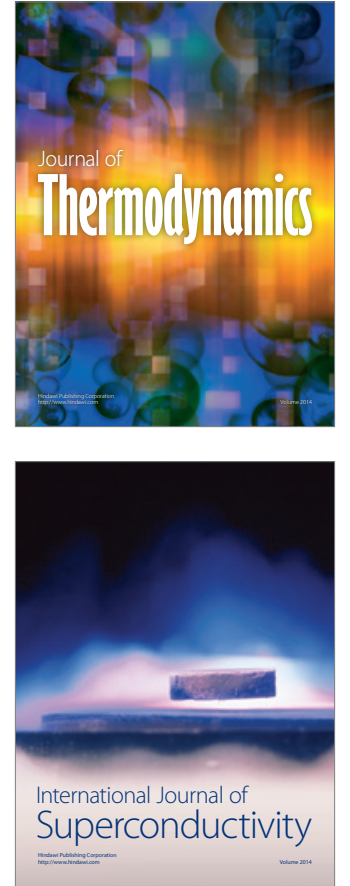
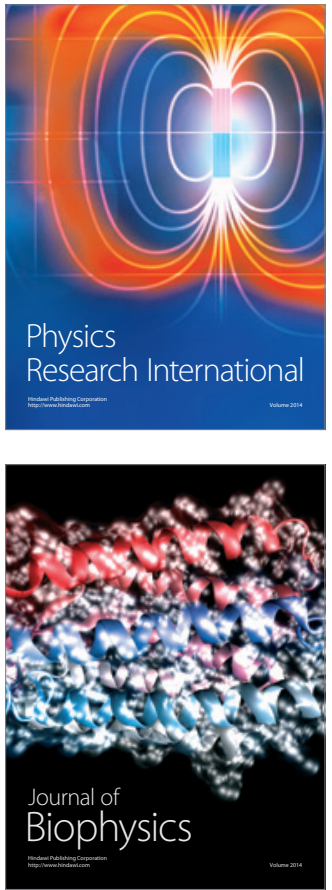
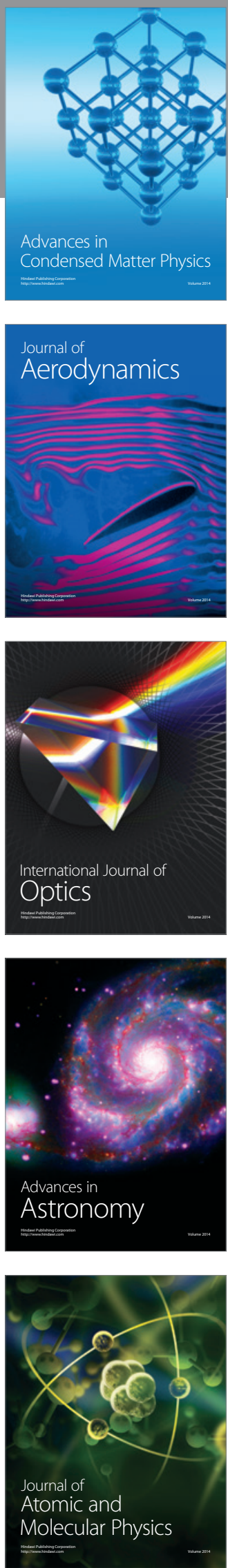